\title{
Tissue oxygen saturation changes and postoperative complications in cardiac surgery: a prospective observational study
}

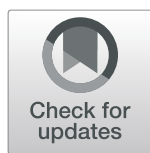

\author{
Sabino Scolletta ${ }^{1 *}$, Federico Franchi ${ }^{1}$, Elisa Damiani ${ }^{2}$, Armando Cennamo ${ }^{1}$, Roberta Domizi ${ }^{2}$, Antonio Meola ${ }^{1}$, \\ Claudia Scorcella ${ }^{2}$, Davide Vanoli ${ }^{1}$, Christopher Münch ${ }^{3}$, Erica Adrario ${ }^{2}$, Luca Marchetti ${ }^{1}$, Fabio Silvio Taccone ${ }^{4}$ and \\ Abele Donati ${ }^{2^{*}}$ (D)
}

\begin{abstract}
Background: Cardiac surgery with extracorporeal circulation (ECC) can induce microvascular dysfunction and tissue hypoperfusion. We hypothesized that the alterations in near-infrared spectroscopy (NIRS)-derived parameters would be associated with post-operative complications in cardiac surgery patients.

Methods: Prospective observational study performed at two University Hospitals. Ninety patients undergoing cardiac surgery with ECC were enrolled. The NIRS sensor was applied on the thenar eminence. A vascular occlusion test (VOT, 3-min ischemia) was performed at baseline (t0), at Intensive Care Unit (ICU) admission (t1), 3 (t2) and 6 (t3) hours later. Baseline tissue oxygen saturation $\left(\mathrm{StO}_{2}\right)$, oxygen extraction rate and microvascular reactivity indices were calculated.

Results: In the first hours after cardiac surgery, $\mathrm{StO}_{2}$ tended to increase (86\% [80-89] at T3 versus 82\% [79-86] at $\mathrm{T} 0, p=\mathrm{ns})$, while both tissue oxygen extraction and microvascular reactivity tended to decrease, as indicated by increasing occlusion slope $(-8.1 \% / \mathrm{min}[-11.2$ to -7$]$ at T3 versus $-11.2 \% / \mathrm{min}[-13.9$ to -7.9$]$ at T0, $p=\mathrm{ns})$ and decreasing recovery slope (1.9\%/sec [1.1-2.9] at T3 versus 3.1\%/sec [2.3-3.9] at T0, $p=$ ns). No substantial differences were found in NIRS-derived variables and their changes over time between patients with complications and those without complications.

Conclusions: Peripheral tissue oxygen extraction and microvascular reactivity were reduced during the first hours after cardiac surgery. NIRS-derived parameters were not able to predict complications in this population of cardiac surgery patients.
\end{abstract}

Keywords: Cardiac surgery, Tissue oxygenation, Near InfraRed spectroscopy, Postoperative complications

\section{Background}

Extracorporeal Circulation (ECC) is associated with significant changes in the physiology of peripheral perfusion $[1,2]$. The main mechanisms by which ECC leads to an impairment in organ perfusion are the activation of inflammatory pathways with consequent endothelial

\footnotetext{
*Correspondence: sabino.scolletta@dbm.unisi.it; a.donati@univpm.it 'Department of Medicine, Surgery and Neuroscience, Anesthesia and Intensive Care Unit, University of Siena, Via Bracci 1, 53100 Siena, Italy ${ }^{2}$ Department of Biomedical Sciences and Public Health, Clinic of Anesthesiology and Intensive Care, AOU Ospedali Riuniti di Ancona, Università Politecnica delle Marche, via Conca 71, 60126 Torrette di Ancona, Ancona, Italy

Full list of author information is available at the end of the article
}

damage, capillary leak [3], interstitial oedema, and impaired microcirculatory blood flow. Consequently, even when global $\mathrm{O}_{2}$ delivery is preserved, a local hypoxia occurs, leading to organ dysfunction that is associated with worse patient outcomes [4]. Near infrared spectroscopy (NIRS) is a non-invasive technique that allows static and dynamic assessments of tissue oxygen saturation in response to ischemic challenge (vascular occlusion test, VOT), thus providing information of peripheral (skeletal muscle) $\mathrm{O}_{2}$ extraction rate and microvascular reactivity $[5,6]$. Alterations in tissue oxygen saturation are associated with higher mortality in patients with sepsis [7]. NIRS monitoring can provide information on the effects

(c) The Author(s). 2019 Open Access This article is distributed under the terms of the Creative Commons Attribution 4.0 International License (http://creativecommons.org/licenses/by/4.0/), which permits unrestricted use, distribution, and 
that therapies may have on tissue perfusion and microcirculation [8]. Only a few single-centre studies investigated the relationship between peripheral NIRS-derived parameters and patients' outcome in cardiac surgery, showing conflicting results and using different devices to assess tissue oxygen saturation [9-15]. Postoperative complications and persistent elevated arterial lactate concentrations were associated with low $\mathrm{StO}_{2}$ after ICU admission [9, 12]. By using a vascular occlusion test (VOT), some studies showed that alterations in the desaturation and reperfusion slopes in the early post-operative phase following cardiac surgery were associated with poor outcome, duration of mechanical ventilation, length of ICU stay, and mortality [11, 13, 15]. Conversely, other studies failed to demonstrate the association between NIRS-derived parameters and outcome [10, 14].

The aim of the present bicentric study was to assess the ability of NIRS-derived parameters to predict postoperative complications in patients undergoing cardiac surgery with ECC.

\section{Methods}

This is a prospective observational study carried out at the University Hospitals of Siena and Ancona, Italy. The local ethics committees of both centres approved the study protocol (Local Ethical Committee of University Hospital of Siena-CEL AOUS, date of approval 23/11/ 2012, and Ethical Committee of Regione Marche-CERM, date of approval 08/11/2013). The patient recruitment started on December 2012 at the University Hospital of Siena and on December 2013 at the University Hospital of Ancona and ended on February 2014 in both centers. Convenience sampling was performed based on the availability of the investigators and/or NIRS device. Written informed consent was obtained from all the included patients the day before surgery. Due to its observational nature, the study was not registered to a specific register. A STrengthening the Reporting of OBservational studies (STROBE) checklist is reported in Additional file 1.

\section{Study protocol}

Adult patients undergoing elective cardiac surgery with ECC were eligible for enrolment in the study. Exclusion criteria were: age $<18$ years, surgery without aortic crossclamping and ECC, surgery with extreme hypothermia or selective brain perfusion, patients undergoing emergency surgery (such as surgery for aortic dissection), and absence of informed consent. The day before surgery (the evening before or the same morning - T0), on admission to the Intensive Care Unit (ICU) (T1), and $3 \mathrm{~h}$ (T2) and $6 \mathrm{~h}$ (T3) after ICU admission, the patients underwent NIRS monitoring and VOT, as described in detail below. No change of therapy occurred between $\mathrm{T} 0$ and the start of surgery.
General haemodynamic, blood gas and laboratory parameters were recorded simultaneously. Any type of post-operative major complication (i.e. cardiac, respiratory, renal, abdominal, neurological, haematological, infectious) occurring during stay in the ICU was also recorded. Major complications, including cardiovascular, respiratory, neurological, renal, infectious, haemorrhagic [16], haematological [17], and abdominal [18] events, were defined accordingly to standard definitions (see details in Additional file 2).

\section{NIRS monitoring}

NIRS is a real-time technology that can measure tissue $\mathrm{O}_{2}$ saturation $\left(\mathrm{StO}_{2}\right)$ continuously using light in the infrared band to detect differences of absorbance between oxy- and deoxy-haemoglobin [19]. The maximum depth of the tissue sample is estimated to equal half the distance between the probe's sending and receiving fibers (probe spacing). A light-scattering calibrator is used to normalize the tissue spectrometer during startup of the system and before each measurement. Sample measurement signals are updated every $3.5 \mathrm{~s}$ [19]. In this study, an InSpectra $\mathrm{StO}_{2}$ Tissue Oxygenation Monitor (model 650; Hutchinson Technology, Hutchinson, MN, USA) was used to measure $\mathrm{StO}_{2}$ at baseline and during a VOT with a $15-\mathrm{mm}$-spaced probe applied on the thenar eminence. The VOT was performed using a pre-defined occlusion time of $3 \mathrm{~min}$. The probe was placed on the hand without the arterial catheter. After a minimum initial 3-min stabilization period (aimed to achieve $\mathrm{StO}_{2}$ excursions within $\pm 3 \%$ over at least $30 \mathrm{~s}$ ), arterial blood flow was obstructed by inflating a sphygmomanometer cuff to a pressure of $50 \mathrm{mmHg}$ above systolic arterial pressure. After $3 \mathrm{~min}$ of occlusion the cuff was rapidly deflated. $\mathrm{StO}_{2}$ was measured continuously until a new stable value was reached. The following parameters were calculated using Inspectra software (InSpectra Analysis Program version 4.00; Hutchinson Technology), as described elsewhere [11, 20] (see Additional file 3 for details):

- baseline $\mathrm{StO}_{2}$

- occlusion slope, an index of tissue $\mathrm{O}_{2}$ extraction rate $(\% / \mathrm{min})$, calculated from the regression line of $\mathrm{StO}_{2}$ decay during the 3 min of blood flow occlusion;

- area of ischemia calculated as area under the occlusion slope curve;

- minimum $\mathrm{StO}_{2}$ obtained at the end of the 3-min ischemia;

- recovery slope calculated from the regression line of $\mathrm{StO}_{2}$ increase during the reperfusion phase of VOT;

- recovery area or area under the curve of the recovery slope;

- maximum $\mathrm{StO}_{2}$ reached during reperfusion; 
- area of hyperaemia or the area under the $\mathrm{StO}_{2}$ curve reflecting, the phase of reactive hyperaemia following VOT.

\section{Standard hemodynamic monitoring}

After admission to the ICU, central venous pressure (CVP) and arterial pressure were evaluated continuously by an invasive intravascular approach. In patients under mechanical ventilation, cardiac output was obtained using the modified carbon dioxide Fick method $\left(\mathrm{mCO}_{2} \mathrm{~F}\right)$. Briefly, The $\mathrm{mCO}_{2} \mathrm{~F}$ method is based on $\mathrm{CO}_{2}$ generation, in which $\mathrm{CO}_{2}$ generation and $\mathrm{O}_{2}$ consumption are always in a linear relationship [21]. Cardiac output obtained using the $\mathrm{mCO}_{2} \mathrm{~F}$ method have showed high accuracy and good reproducibility, to respect classic thermodilution technique [22]. We used the standard formula: $\mathrm{CO}=\mathrm{VCO}_{2} / \mathrm{R}^{*} \mathrm{da}$ $\mathrm{vO}_{2}$, where $\mathrm{VCO}_{2}$ is the carbon dioxide production $(\mathrm{ml} /$ $\mathrm{min}$ ) provided by capnometry, $\mathrm{R}$ the Respiratory Exchange Rate, and da- $-\mathrm{vO}_{2}$ the arterial-venous oxygen difference. An assumed value of the Respiratory Exchange Ratio equal to 0.9 was used for all patients [22].. The cardiac index (CI) was obtained by dividing cardiac output by body surface area. Systemic vascular resistance index (SVRI), oxygen delivery index $\left(\mathrm{DO}_{2} \mathrm{I}\right)$ and oxygen extraction ratio $\left(\mathrm{O}_{2} \mathrm{ER}\right)$ were calculated with standard formulas. Arterial and venous gas analyses were performed at $\mathrm{T} 0, \mathrm{~T} 1, \mathrm{~T} 2$ and $\mathrm{T} 3$.

\section{Anaesthesia, cardiopulmonary bypass, and myocardial protection}

Anaesthesia was induced with $1 \mu \mathrm{g}^{*} \mathrm{~kg}^{-1}$ fentanyl, 0.2 $m g^{*} \mathrm{~kg}^{-1}$ midazolam or $2 \mathrm{mg}^{*} \mathrm{~kg}^{-1}$ propofol, and 0.8 $\mathrm{mg}^{*} \mathrm{~kg}^{-1}$ rocuronium; $2-2.5 \%$ sevoflurane and continuous IV infusion of remifentanil $\left(0.1-0.5 \mu \mathrm{g}^{*} \mathrm{~kg}^{-1 *}\right.$ min) were used for maintenance. At the beginning of cardiopulmonary bypass $(\mathrm{CPB})$, sevoflurane was discontinued and replaced with propofol at a maximum infusion rate of $4 \mathrm{mg}^{*} \mathrm{~kg}^{-1}$ "h IV and a bolus of $5 \mathrm{mg}^{*} \mathrm{~kg}^{-1}$ thiopental IV was administered.

ECC was instituted with a roller pump (Terumo Perfusion System 1, Terumo Corporation, Tokyo, Japan), or a centrifugal pump (Revolution Sorin, MedicalExpo, London, UK) and a hollow-fibre oxygenator (Capiox RX 25; Terumo Corporation, Tokyo, Japan) primed with $1200-1500 \mathrm{~mL}$ buffered crystalloid solution. During ECC, haemoglobin levels were maintained between 8 and $9 \mathrm{~g}^{*} \mathrm{dl}^{-1}$ (haematocrit about 24\%). Similar protocols for the ECC were used in the two centers. Myocardial protection was obtained with St. Thomas blood cardioplegia solution or Custodiol solution [23]. The pump flow rate was calculated based on the patient BSA, as follows: Flow $\left(\mathrm{L}^{*} \mathrm{~min}^{-1 *} \mathrm{~m} 2\right)=\mathrm{BSA} * 2.4$. Moderate systemic hypothermia $\left(32-34^{\circ} \mathrm{C}\right)$ was maintained using a heat exchanger connected with ECC machine and $\alpha$-stat acid-base management was applied.
The patients were weaned off ECC when rectal temperature had reached $34{ }^{\circ} \mathrm{C}$. In the ICU, patients were weaned off mechanical ventilation as soon as they were awake and breathing faster than the ventilator set rate and when the following criteria were met: patient obeying commands; stable and adequate haemodynamics; no significant arrhythmia; core temperature of higher than $36^{\circ} \mathrm{C}$; chest tube drainage of less than 100 $\mathrm{mL}^{*} \mathrm{~h}^{-1}$ for 2 consecutive hours; diuresis of more than 1 $\mathrm{mL}^{*} \mathrm{~kg}^{-1}$ per hour; arterial carbon dioxide pressure $\left(\mathrm{PaCO}_{2}\right)$ of less than $50 \mathrm{mmHg}$; arterial $\mathrm{O}_{2}$ pressure $\left(\mathrm{PaO}_{2}\right)$ of more than $70 \mathrm{mmHg}$; and $\mathrm{O}_{2}$ saturation as measured by pulse oxymetry of $92 \%$ or higher with the patient breathing less than $50 \% \mathrm{O}_{2}$.

\section{Statistical analysis}

Statistical analysis was performed using statistical software (SPSS 21.0, SPSS, Chicago, Illinois, and Prism 6.0, GraphPad Software, San Diego, California). The ShapiroWilk test was used to test normality of distribution. Data was presented as median [interquartile range], number or percentage, as appropriate. The chi square test was used for comparison of categorical variables. Since the data were not normally distributed, the Mann Whitney $\mathrm{U}$ test or the Friedman test with Dunn's post hoc test for multiple comparisons were used to evaluate differences between groups or over time, as appropriate. Delta values of NIRS-derived variables were also calculated and a two-way analysis of variance (2-way ANOVA) was used to evaluate differences between the two groups at different time points. Binary logistic regression analyses were performed to test the association of changes in NIRS-derived variables and post-operative complications by adjusting for relevant clinical parameters (we constructed one model for each NIRS-derived parameter). A Spearman's rho was calculated to assess correlations between variables. In order to address the multiple testing problem, the Bonferroni correction was applied to adjust the alpha level of significance. The corrected level of significance after Bonferroni correction for multiple testing was arbitrarily set at $\alpha=0.001$. Therefore, an unadjusted $p$ value $<0.001$ was considered to show statistical significance (all reported $p$ values are unadjusted).

\section{Results}

A convenience sample of 90 patients was studied. Sixty patients were enrolled at the University Hospital of Siena and 30 at the University Hospital of Ancona (Fig. 1). Mean age was 70 [64-76] years and 59 patients (65.6\%) were male. The type of surgery was: aortic/mitral valve replacement in 52 patients (58\%), coronary artery bypass graft in 17 patients (19\%), combined procedures in 17 cases $(19 \%)$ and other procedures in 4 cases $(4 \%)$. The logistic Euroscore of the patients was $8.2 \pm 7.6$, and the 


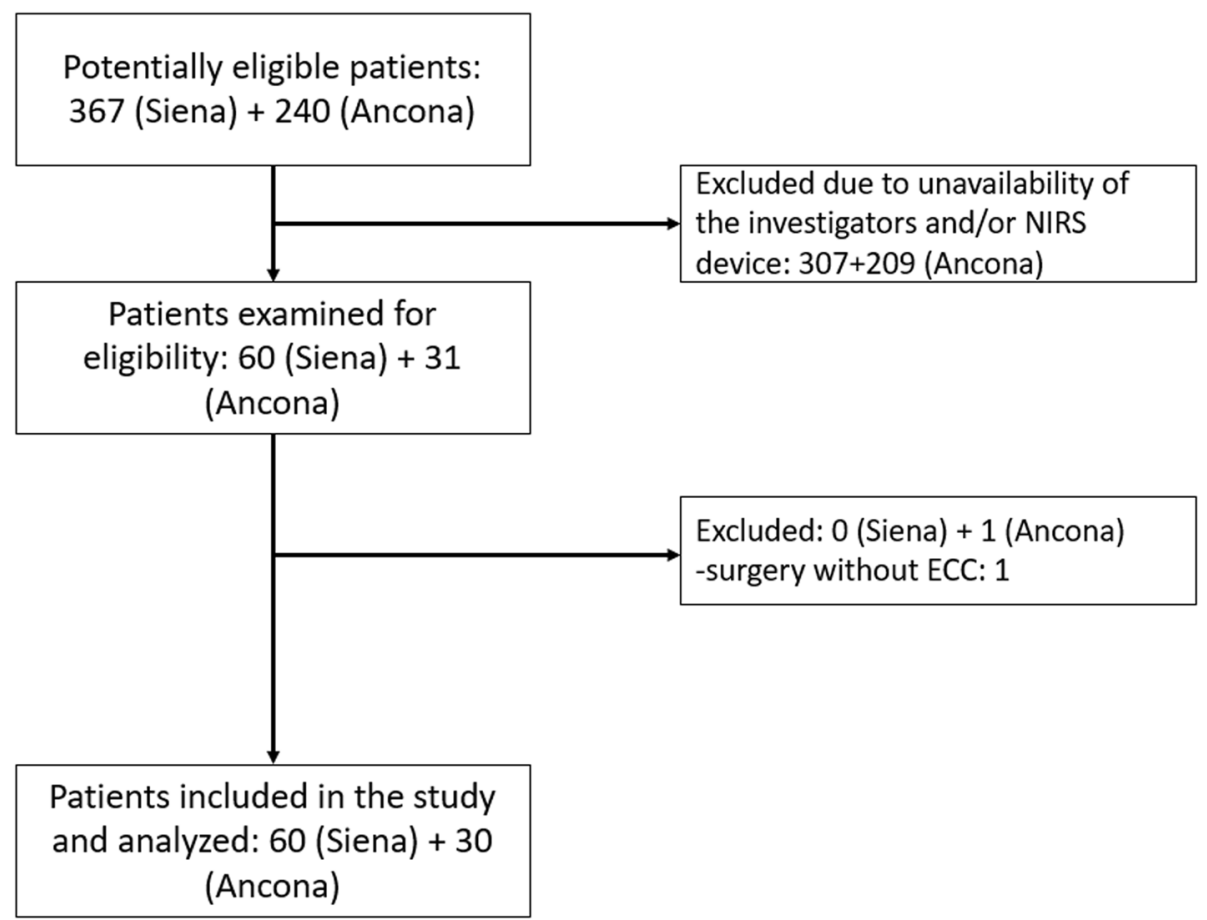

Fig. 1 Study flow chart

length of stay in the ICU was 2 [1-5] days. Thirty-nine patients (43\%) developed at least one post-operative complication during their ICU stay. Cardiovascular complications occurred in twenty-seven $(30 \%)$ patients, respiratory in $13(14 \%)$, renal in $9(10 \%)$, abdominal in 3 (3\%), neurological in 6 (7\%), haematological (including haemorrhagic) in $3(3 \%)$, and infectious in $4(4 \%)$ patients. Patients with and without complications did not significantly differ in age, gender, presence of comorbidities, duration of ECC or clamping, worst values of mean arterial pressure (MAP), haematocrit, haemoglobin ( $\mathrm{Hb})$, $\mathrm{SvO}_{2}$, lactate or blood glucose during ECC (Table 1). Variations in hemodynamic parameters are reported in Table 2: no significant differences were found between patients with complications and those without complications.

\section{NIRS-derived parameters and outcome}

In the overall population, $\mathrm{StO}_{2}$ tended to increase above baseline values $(86 \%$ [80-89] at T3 versus $82 \%$ [79-86] at T0, $p=0.003$ ). Muscle tissue $\mathrm{O}_{2}$ extraction rate tended to be reduced in the first $6 \mathrm{~h}$ after surgery, as indicated by a flatter occlusion slope $(-8.1 \% / \mathrm{min}[-11.2$ to -7$]$ at $\mathrm{T} 3$ versus $-11.2 \% / \mathrm{min}[-13.9$ to -7.9$]$ at $\mathrm{T} 0, p=0.003)$, smaller area of ischemia (37.4 [31.8-46.5] at T3 versus 47.2 [36.5-60.6] at T0, $p=0.002)$ and higher minimum $\mathrm{StO}_{2}$ after 3-min flow occlusion (56\% [48-65] at T3 versus $47 \%$ [39-55] at T0, $p<0.001)$. Recovery slope was reduced in the first $6 \mathrm{~h}$ in ICU $(1.9 \% / \mathrm{sec}[1.1-2.9]$ at T3 versus $3.1 \% / \mathrm{sec}[2.3-3.9]$ at $\mathrm{T} 0, p=0.001)$, while recovery area, area of hyperaemia and maximum $\mathrm{StO}_{2}$ during recovery were similar to baseline values at T3. Comparison of patients with and without post-operative complications is shown in Fig. 2. No significant differences were found in the variations of NIRS-derived parameters between the two groups (Fig. 2, please see Additional file 4 for comparisons of delta values). Patients with a worsening of NIRSderived parameters at T3 did not show higher incidence of complications (Additional file 5). Logistic regression analyses (adjusting for age, lactate, MAP, $\mathrm{Hb}$ and $\mathrm{HR}$ ) showed no association of delta values of $\mathrm{StO} 2$ (odds ratio 1.068 [95\% confidence interval 0.994-1.147], $p=0.073$ ), occlusion slope (1.062 [0.963-1.170], $p=0.226$ ), recovery slope (1.237 [0.929-1.646], $p=0.146)$ or area of hyperaemia (1.016 [0.962-1.072], $p=0.575)$ at T3 and postoperative complications.

Similar results were obtained comparing patients with cardiovascular complications $(n=26,29 \%)$, versus those with other complications $(n=13,14 \%)$ versus those without complications ( $n=51,57 \%)$ (Additional file 6). Correlation analyses between NIRS-derived parameters at ICU systemic admission and intraoperative parameters are reported in Additional file 7. Correlations between NIRSderived parameters and hemodynamic parameters in pooled data are reported in Additional file 8.

\section{Discussion}

In the present study of 90 patients undergoing cardiac surgery with ECC, NIRS monitoring at the thenar eminence 
Table 1 General data and intraoperative parameters in patients with or without post-operative complications

\begin{tabular}{|c|c|c|c|}
\hline & No Complications $(n=51)$ & $\begin{array}{l}\text { Complications } \\
(n=39)\end{array}$ & $p$ \\
\hline Age (years) & $72[63-76]$ & $70[65-75]$ & 0.654 \\
\hline Gender ( $\mathrm{n}[\%$ of males]) & 30 [59\%] & 29 [74\%] & 0.174 \\
\hline \multicolumn{4}{|l|}{ Comorbidities (n [\%]) } \\
\hline Arterial hypertension & 42 [82\%] & 27 [69\%] & 0.208 \\
\hline Dyslipidemia & 19 [37\%] & 12 [31\%] & 0.655 \\
\hline Coronary Artery Disease & 9 [18\%] & 12 [31\%] & 0.208 \\
\hline Diabetes mellitus & 13 [25\%] & $6[15 \%]$ & 0.303 \\
\hline Chronic cardiac failure & 9 [18\%] & $3[8 \%]$ & 0.219 \\
\hline Chronic renal failure & $4[8 \%]$ & 8 [20\%] & 0.117 \\
\hline Chronic Obstructive Pulmonary Disease & $5[10 \%]$ & 4 [10\%] & 0.999 \\
\hline Duration of ECC (min) & $126[96-167]$ & $116[81-166]$ & 0.523 \\
\hline Duration of clamping (min) & $100[68-126]$ & 95 [60-129] & 0.631 \\
\hline \multicolumn{4}{|l|}{ Worst parameters during ECC } \\
\hline $\operatorname{Min} \mathrm{MAP}(\mathrm{mmHg})$ & $60[50-65]$ & $55[50-61]$ & 0.158 \\
\hline Min haematocrit (\%) & 25 [22-28] & $24[21-27]$ & 0.771 \\
\hline Min haemoglobin ( $g / d L)$ & $8.0[7.0-8.8]$ & $7.9[6.8-9.0]$ & 0.924 \\
\hline Min $\mathrm{SvO}_{2}(\%)$ & $82[80-86]$ & $81[78-84]$ & 0.227 \\
\hline Max lactate (mmol/L) & $2.2[1.5-3.1]$ & 2.4 [1.9-3.3] & 0.337 \\
\hline Max blood glucose (mg/dL) & 168 [136-186] & 156 [137-192] & 0.918 \\
\hline
\end{tabular}

Data are expressed as median [1st-3rd quartiles] or numbers and percentages

ECC extracorporeal circulation, MAP mean arterial pressure, $\mathrm{SvO}_{2}$ venous oxygen saturation

with VOT detected a significant reduction in skeletal muscle microvascular reactivity following surgery, together with a decrease in tissue $\mathrm{O}_{2}$ extraction rate, which did not recover in the first $6 \mathrm{~h}$ after the operation. We failed to detect an association between NIRS-derived parameters and patient outcome since patients with postoperative complications showed similar variations as those without complications.

It is well known that cardiac surgery with ECC can induce a complex inflammatory response. This can be due to multiple factors, including surgical trauma, haemodilution, ischemia/reperfusion injury, hypothermia and exposure of blood to non-physiological surfaces $[1,3]$. The mechanisms involve the following: the release of cytokines, complement activation, leukocyte activation with endothelial adhesion, an increased production of $\mathrm{O}_{2}$ free radicals, the release of inflammatory mediators including endothelin, and the deregulation of the nitric oxide pathway [24]. Under these conditions, systemic haemodynamic parameters and markers of global oxygenation, such as central venous $\mathrm{O}_{2}$ saturation or arterial lactate, may not be early predictors of tissue hypoperfusion. Although increased lactate levels are related to morbidity and mortality in different patient groups [25], they lack sensitivity and specificity in representing tissue perfusion and may not be sufficient to detect the early impairment of tissue oxygenation [26-28]. The pathological mechanism triggered by the ECC showed strong similarities to those seen during sepsis, potentially leading to impaired microcirculatory perfusion and tissue hypoxia. Studies using sublingual videomicroscopy have shown alterations in microvascular perfusion, which may persist for $24 \mathrm{~h}$ after surgery [29] and may occur irrespective of changes in systemic haemodynamics [30]. Bauer et al. showed an increased number of rolling leukocytes in the sublingual microcirculation during $\mathrm{CPB}$, which persisted $1 \mathrm{~h}$ after the termination of CPB [31].

NIRS monitoring in conjunction with VOT enables us to estimate peripheral tissue oxygen saturation and microvascular reactivity by evaluating variations in $\mathrm{StO}_{2}$ during a brief ischemia/reperfusion test [5]. In several studies using this technology, patients undergoing cardiac surgery showed impaired microvascular reactivity, although conflicting data exists regarding the time needed for recovery to the baseline microvascular state and the relationship with outcome. Smith et al. showed that during $\mathrm{CPB}$, the reperfusion slope decreased as a function of $\mathrm{CPB}$ duration, returning to baseline values in all patients within $1 \mathrm{~h}$ of the termination of CPB [32]. In another study by Morel et al., $\mathrm{StO}_{2}$ and reperfusion slope both declined after CPB but recovered to baseline values after $12 \mathrm{~h}$ [10]. Furthermore, these transient 
Table 2 Variations in haemodynamic parameters in patients with $(n=39)$ or without $(n=51)$ post-operative complications

\begin{tabular}{|c|c|c|c|c|}
\hline & to & $\mathrm{t} 1$ & $\mathrm{t} 2$ & t3 \\
\hline \multicolumn{5}{|l|}{$\mathrm{MAP}(\mathrm{mmHg})$} \\
\hline All & 91 [83-102] & $79[72-86]^{*}$ & $80[73-91]^{*}$ & $82[73-92]^{*}$ \\
\hline No complications & 93 [82-102] & 83 [73-90] & 84 [77-95] & 84 [74-92] \\
\hline Complications & 90 [87-102] & $77[68-85]^{*}$ & $75[71-87]^{*}$ & 78 [72-91] \\
\hline \multicolumn{5}{|l|}{ Heart Rate (bpm) } \\
\hline All & 68 [60-75] & $85[79-91]^{*}$ & $87[80-90]^{*}$ & $86[80-90]^{*}$ \\
\hline No complications & 68 [57-77] & $86[80-90]^{*}$ & $86[80-90]^{*}$ & $86[79-90]^{*}$ \\
\hline Complications & 70 [61-74] & $86[80-93]^{*}$ & $87[79-90]^{*}$ & $87[80-90]^{*}$ \\
\hline \multicolumn{5}{|l|}{ CVP $(\mathrm{mmHg})$} \\
\hline All & NA & 10 [7-12] & 9 [7-12] & 8 [7-11] \\
\hline No complications & NA & 9 [7-12] & 8 [7-12] & $8[7-11]$ \\
\hline Complications & NA & 10 [8-13] & 10 [8-12] & 9 [7-12] \\
\hline \multicolumn{5}{|l|}{$\mathrm{Hb}(\mathrm{g} / \mathrm{dL})$} \\
\hline All & 14.1 [13.2-15.3] & $11.5[10.3-12.9]^{*}$ & $12.5[11.5-13.6]^{*}$ & $12.4[11.2-13.5]^{*}$ \\
\hline No complications & $14.1[13.2-15.6]$ & $12[10.5-13.1]^{*}$ & 12.6 [11.6-13.5] & $12.5[11.1-13.6]^{*}$ \\
\hline Complications & 14.4 [13.2-15] & $11.1[10.2-11.8]^{*}$ & 11.7 [10.6-13.2] & 12 [10.4-12.5] \\
\hline \multicolumn{5}{|l|}{ Lactate $(\mathrm{mmol} / \mathrm{l})$} \\
\hline All & $1.4[1.1-1.9]$ & $2.3[1.5-3.3]^{*}$ & $2.0[1.4-2.9]^{*}$ & $1.9[1.6-2.8]^{*}$ \\
\hline No complications & 1.5 [1.2-2] & $2.0[1.3-2.6]$ & $1.8[1.3-2.7]$ & $1.9[1.4-2.6]^{*}$ \\
\hline Complications & $1.3[1.0-1.6]$ & $2.0[1.4-4.1]$ & $1.8[1.4-3.5]$ & $1.9[1.4-3.2]$ \\
\hline \multicolumn{5}{|l|}{$\mathrm{SvO}_{2}(\%)$} \\
\hline All & NA & 72 [66-77] & 70 [63-75] & 71 [64-76] \\
\hline No complications & NA & 71 [67-75] & 71 [65-75] & 70 [63-76] \\
\hline Complications & NA & 74 [63-81] & 69 [60-76] & 72 [65-81] \\
\hline \multicolumn{5}{|l|}{$\mathrm{Cl}\left(\mathrm{L} / \mathrm{min} / \mathrm{m}^{2}\right)(\mathrm{n})$} \\
\hline All & NA & $2.1[1.7-2.7](90)$ & $1.9[1.5-2.3](87)$ & $2.2[1.9-2.5](66)$ \\
\hline No complications & NA & $2.1[1.8-2.4](51)$ & $1.9[1.6-2.4](49)$ & $2.1[1.8-2.5](34)$ \\
\hline Complications & NA & $2.5[1.6-3.3](39)$ & $2.0[1.5-2.6](38)$ & $2.2[1.9-3.1](32)$ \\
\hline \multicolumn{5}{|l|}{ SVRI $\left(d y n^{*} s / \mathrm{cm}^{5 *} \mathrm{~m}^{2}\right)$} \\
\hline All & NA & 2625 [2250-3415] (90) & 3266 [2302-3903] (87) & 2639 [2044-3193] (66) \\
\hline No complications & NA & 2732 [2195-3284] (51) & 3274 [2426-3832] (49) & 2747 [2077-3202] (34) \\
\hline Complications & NA & 2364 [1958-3090] (39) & 2939 [2282-3813] (38) & 2643 [1772-3197] (32) \\
\hline \multicolumn{5}{|l|}{$\mathrm{DO}_{2} \mathrm{I}\left(\mathrm{ml} / \mathrm{min} / \mathrm{m}^{2}\right)$} \\
\hline All & NA & 337 [231-382] (90) & 308 [233-378] (87) & 347 [282-425] (66) \\
\hline No complications & NA & 337 [257-377] (51) & 335 [273-381] (49) & 343 [288-409] (34) \\
\hline Complications & NA & 341 [218-443] (39) & 306 [241-393] (38) & 344 [281-436] (32) \\
\hline \multicolumn{5}{|l|}{$\mathrm{O}_{2} \mathrm{ER}(\%)$} \\
\hline All & NA & 26 [22-36] (90) & 30 [26-37] (87) & 29 [25-35] (66) \\
\hline No complications & NA & 26 [23-31] (51) & 28 [26-33] (49) & 28 [25-35] (34) \\
\hline Complications & NA & 27 [22-38] (39) & 35 [27-42] (38) & 31 [25-35] (32) \\
\hline \multicolumn{5}{|l|}{ Temperature $\left({ }^{\circ} \mathrm{C}\right)$} \\
\hline All & NA & $35.2[35-36]$ & $36.0[35.5-36.8]^{*}$ & $36.5[36.0-37.1]^{*}$ \\
\hline No complications & NA & $35.2[35-36]$ & $36.0[35.5-36.9]^{*}$ & $36.5[36-37]^{*}$ \\
\hline Complications & NA & $35.5[35-36]$ & $36.2[35.7-36.8]^{*}$ & $36.7[36.2-37.3]^{*}$ \\
\hline
\end{tabular}


Table 2 Variations in haemodynamic parameters in patients with $(n=39)$ or without $(n=51)$ post-operative complications (Continued)

\begin{tabular}{lllll}
\hline & t0 & t1 & t2 & t3 \\
\hline Mechanical ventilation (n [\%]) & & & & $66[73 \%]$ \\
All & - & $90[100 \%]$ & $87[97 \%]$ & $34[67 \%]$ \\
No complications & - & $51[100 \%]$ & $49[96 \%]$ & $32[82 \%]$ \\
Complications & - & $39[100 \%]$ & $38[97 \%]$ & $33[37 \%]$ \\
Vasopressors (n, \%) & & & $15[29 \%]$ \\
All & - & $34[38 \%]$ & $37[41 \%]$ & $18[46 \%]$ \\
No complications & - & $16[31 \%]$ & $16[31 \%]$ & $21[54 \%]$ \\
Complications & - & $18[46 \%]$ & & $26[29 \%]$ \\
Inotropic drugs (n, \%) & - & $24[27 \%]$ & $12[24 \%]$ & $11[22 \%]$ \\
All & - & $12[24 \%]$ & $13[32 \%]$ & $15[37 \%]$ \\
No complications & - & $12[30 \%]$ & \\
Complications & - & & $25 \%]$ \\
\hline
\end{tabular}

Data are expressed as median [1st-3rd quartiles] or numbers and percentages

${ }^{*} p<0.001$ versus baseline, a Friedman test with Dunn's post hoc test for multiple comparisons was used to evaluate changes over time in each group (according to the Bonferroni correction, an unadjusted $p<0.001$ was used to indicate statistical significance)

to baseline, $t 1$ post surgery, $t 2$ three hours after intensive care unit admission, $t 3$ six hours after intensive care unit admission, MAP mean arterial pressure, CVP central venous pressure, $\mathrm{Hb}$ haemoglobin, $\mathrm{SvO}_{2}$ venous oxygen saturation, $\mathrm{Cl}$ cardiac index, $\mathrm{SVRl}$ systemic vascular resistance index, $\mathrm{DO}_{2} \mathrm{I}$ oxygen delivery index, $\mathrm{O}_{2} E R$ oxygen extraction rate

changes were not correlated with the patients' outcome. We found a similar trend in the recovery slope at the first $6 \mathrm{~h}$ of the study; however, we did not collect NIRS parameters after $6 \mathrm{~h}$. There are two main differences between our study and that by Morel et al. [10]. Firstly, we used major complications as variables of outcome, instead of using the Sequential Organ Failure Assessment score. Secondly, the duration of VOT was different in the two studies: we applied a three minute time targeted VOT, while Morel et al. maintained the VOT until the $\mathrm{StO}_{2}$ value reached $40 \%$. However, despite these differences, our results were consistent with those showed by Morel [10]. Kim et al. demonstrated that the reperfusion slope largely recovered on the first day after surgery in patients without complications, while it remained altered in those with complications [12]. In the present study, the recovery slope remained reduced in patients for $6 \mathrm{~h}$ after admission to the ICU, indicating a persistent decrease in microvascular reactivity. However, we were unable to detect more severe alterations or delayed recovery of this parameter among patients with postoperative complications in such a short monitoring period. There may be several explanations for these discrepant results. First, we performed 3-min blood flow occlusion in all patients, instead of using a target $\mathrm{StO}_{2}$, and this may have produced different degrees of ischemia. It has been shown that $\mathrm{StO}_{2}$ recovery rate depends on the minimum $\mathrm{StO}_{2}$ reached after $3 \mathrm{~min}$ of blood flow occlusion; i.e. the velocity of reperfusion and the degree of hyperaemia are related to the degree of ischemia [33]. Second, it is possible that the monitoring period in this study was too short to detect differences for predicting post-operative morbidity.
Kopp et al. showed that $\mathrm{StO}_{2}$ was reduced after cardiac surgery and that the minimum value of $\mathrm{StO}_{2}$ in the first hours after the operation was predictive of delayed lactate clearance [13]. However, in a similar patient population other authors have found a transient increase in $\mathrm{StO}_{2}$ after surgery [34]. $\mathrm{StO}_{2}$ reflects the balance between regional $\mathrm{O}_{2}$ delivery and consumption [5]. In our study, in the first $6 \mathrm{~h}$ after surgery, we observed an increase in $\mathrm{StO}_{2}$, a slower desaturation rate (flatter occlusion slope), smaller area of ischemia, and increased $\mathrm{StO}_{2}$ values (nadir) during 3-min of ischemia, but no difference was seen between patients with and without complications. Taken together, these findings suggest a reduction in regional $\mathrm{O}_{2}$ consumption, despite systemic $\mathrm{O}_{2} \mathrm{ER}$ resulting in the normal range in both groups of patients. Several factors may influence $\mathrm{O}_{2}$ extraction and consumption in skeletal muscle, including the administration of sedative or vasopressor agents, a residual neuromuscular blockade in the first post-operative hours, and variations in body temperature $[4,35]$.

Our study has several limitations. First of all, we studied a convenience sample of 90 patients without preliminary statistical calculation of the required sample size. Based on previous studies, we retrospectively calculated that the inclusion of a total of 96 patients was required to show a difference in the reperfusion slope between patients with, and those without, post-operative complications (calculated Cohen's $\mathrm{d}=0.58$ [12]) with a power of $80 \%$ and an alpha error of 0.05 . Therefore, our study may be slightly underpowered, although it is highly unlikely that the inclusion of 6 additional patients would have changed our results substantially. Second, by applying NIRS to 


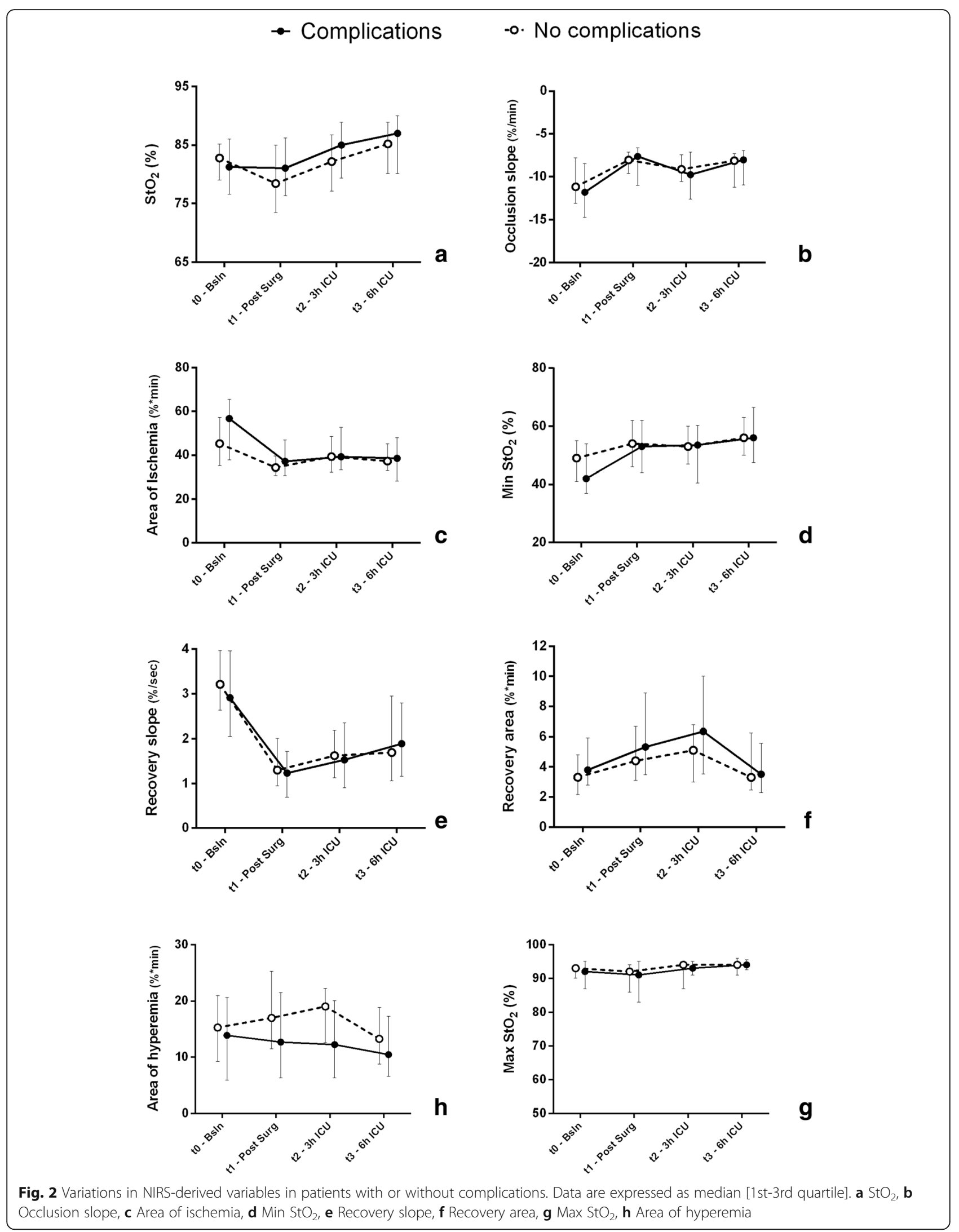


the thenar eminence, we evaluated tissue oxygen saturation and microvascular reactivity in a peripheral tissue. We cannot determine whether similar alterations were induced in microvascular beds of the splanchnic organs. We used the thenar eminence because the thickness of the adipose tissue covering this muscle is small. Although tissue oedema can increase the thickness of the subcutaneous layer, this hardly happens in a period of time as short as that of our study [20]. Third, since the degree of neuromuscular blockade was not measured in our patients, it is possible that the reduction in muscle $\mathrm{O}_{2}$ extraction rate may at least partly depend on a residual neuromuscular blockade in the early postoperative period. Moreover, the use of vasopressors, inotropes and transfusions, as well as the perioperative fluid balance, could affect peripheral tissue oxygen supply and utilization. Unfortunately however, we could not evaluate the potential influence of these treatments because data on vasoactive dosage or fluid and transfusion requirements were not collected. Similarly, we were unable to evaluate the impact of different ECC protocols (e.g the use of roller versus centrifugal pump). Fourth, we did not perform intraoperative NIRS measurements that could provide information about earlier variations in tissue oxygen saturation and microvascular function and their impact on clinical outcomes. Fifth, a target $\mathrm{StO}_{2}$ for VOT, instead of a pre-defined occlusion time, may have been more appropriate for standardizing the degree of ischemia and the hyperaemic phase [33]. Furthermore, we did not collect the tissue haemoglobin index, usually used to calculate the muscle oxygen consumption $\left(\mathrm{NirVO}_{2}\right)$; therefore, we could not evaluate the impact of variations of the $\mathrm{NirVO}_{2}$ on the outcome. Sixth, we did not evaluate the potential role of intraoperative complications or risky events (e.g., arterial hypotension, haemorrhage, low cardiac output) on NIRS parameter and their relationship with the outcome. Again, the tissue spectrometer (InSpectra Model 650) used in the present study, and the company that produces it (Hutchinson Technology, Hutchinson, MN, USA) are actually out of the market. Similar devices using near infrared spectroscopy exist that can provide tissue $\mathrm{O}_{2}$ saturation. Nonetheless, it must be recognized that differences in the proprietary algorithms of different NIRS devices make the comparisons between studies difficult.

Finally, we performed multiple comparisons on a number of variables, thus our analysis may be affected by bias due to multiple-testing and data coupling problems. Indeed, the slopes for the decreasing and increasing $\mathrm{StO} 2$ during the VOT are not independent from the areas that are defined by the slopes; therefore, a data coupling may occur. Nonetheless, we applied the Bonferroni correction to enhance the robustness of our results, and NIRS variables were included individually in separate logistic regression models in order to avoid the problem of collinearity. Even if the study is observational, not having registered it in any public register, the designs and statistical analyses are unverifiable.

\section{Conclusions}

In patients undergoing cardiac surgery with ECC, thenar NIRS monitoring in conjunction with VOT in the first 6 $\mathrm{h}$ after the operation showed a reduction in peripheral tissue $\mathrm{O}_{2}$ extraction and microvascular reactivity. NIRSderived parameters were not able to predict postoperative complications in this population of cardiac surgery patients.

\section{Supplementary information}

Supplementary information accompanies this paper at https://doi.org/10. 1186/s12871-019-0905-5.
Additional file 1. STROBE checklist.
Additional file 2. Definitions of post-operative complications.
Additional file 3. NIRS monitoring technique.
Additional file 4. Comparisons of delta values of NIRS-derived variables between patients with complications and those without complications. Data are expressed as mean \pm standard deviation. Repeated measures 2- way ANOVA with Bonferroni post hoc test.

Additional file 5. Incidence of post-operative complications among patients with a worsening in NIRS-derived variables at T3.

Additional file 6. Variations in NIRS derived parameters in patients with post-operative cardiac complications $(n=26)$ versus those with other complications ( $n=13$ ) versus those without complications $(n=51)$.

Additional file 7. Correlation analyses between NIRS-derived parameters at T1 (time of admission to the ICU) and intraoperative parameters.

Additional file 8. Correlation analyses between NIRS-derived parameters and hemodynamic parameters in pooled data.

\section{Abbreviations}

BSA: Body surface area; Cl: Cardiac index; CPB: Cardiopulmonary bypass; CVP: Central venous pressure; $\mathrm{DO}_{2}$ l: Systemic oxygen delivery index; ECC: Extracorporeal circulation; Hb: Haemoglobin; ICU: Intensive care unit; MAP: Mean arterial pressure; NIRS: Near infrared spectroscopy; $\mathrm{NirV}_{2}$ : Muscle oxygen consumption; $\mathrm{O}_{2}$ : Oxygen; $\mathrm{O}_{2}$ ER: Oxygen extraction rati; $\mathrm{PaCO}_{2}$ : Arterial carbon dioxide pressure; $\mathrm{PaO}_{2}$ : Arterial oxygen pressure; $\mathrm{StO}_{2}$ : Tissue oxygen saturation; $\mathrm{SvO}_{2}$ : Venous oxygen saturation; SVRI: Systemic vascular resistance index; VOT: Vascular occlusion test

\section{Acknowledgments}

None.

\section{Author contributions}

SS and AD designed the study, interpreted the results and revised the manuscript for important intellectual content. FF and ED analyzed and interpreted the data and drafted the manuscript. AC, RD, AM, SC, DV collected and analyzed the data. CM, LM, EA, FST supervised the study, contributed to the interpretation of the results and revised the manuscript critically for important intellectual content. All authors read the manuscript and gave final approval of the version to be published. All authors agree to be accountable for all aspects of the work in ensuring that questions related to the accuracy or integrity of any part of the work are appropriately investigated and resolved.

Funding

None.

Availability of data and materials

The datasets generated and analysed during the current study are available from the corresponding author on reasonable request. 


\section{Ethics approval and consent to participate}

The study was approved by the local ethics committee of University Hospital of Siena-CEL AOUS (date of approval 23/11/2012) and by the ethics committee of Regione Marche-CERM for the University Hospital of Ancona (date of approval 08/11/2013). Written informed consent was obtained from all patients the day before surgery. Due to its pure observational nature, the study was not registered to a specific register.

\section{Consent for publication}

Not applicable.

\section{Competing interests}

The authors declare that they have no competing interests.

\section{Author details}

Department of Medicine, Surgery and Neuroscience, Anesthesia and Intensive Care Unit, University of Siena, Via Bracci 1, 53100 Siena, Italy. ${ }^{2}$ Department of Biomedical Sciences and Public Health, Clinic of Anesthesiology and Intensive Care, AOU Ospedali Riuniti di Ancona, Università Politecnica delle Marche, via Conca 71, 60126 Torrette di Ancona, Ancona, Italy. ${ }^{3}$ Cardiac Anesthesia and Intensive Care Unit, AOU Ospedali Riuniti di Ancona, via Tronto 10/a, 60126 Torrette di Ancona, Ancona, Italy. ${ }^{4}$ Department of Intensive Care, Université Libre de Bruxelles, Hospital Erasme, Route de Lennik, 808 -, 1070 Brussels, Belgium.

\section{Received: 18 January 2019 Accepted: 6 December 2019}

Published online: 16 December 2019

\section{References}

1. Esper SA, Subramaniam K, Tanaka KA. Pathophysiology of cardiopulmonary by-pass: current strategies for the prevention and treatment of anemia, coagulopathy, and organ dysfunction. Semin Cardiothorac Vasc Anesth. 2014;18:161-76.

2. De Somer F. End-organ protection in cardiac surgery. Minerva Anestesiol. 2013;79:285-93.

3. Butler J, Rocker GM, Westaby S. Inflammatory response to cardiopulmonary bypass. Ann Thorac Surg. 1993:55:552-9.

4. Mandak J, Pojar M, Cibicek N, Lonsky V, Palicka V, Kakrdova D, et al. Impact of cardiopulmonary bypass on peripheral tissue metabolism and microvascular blood flow. Perfusion. 2008;23:339-46.

5. Gomez H, Torres A, Polanco P, Kim HK, Zenker S, Puyana JC, et al. Use of non-invasive NIRS during a vascular occlusion test to assess dynamic tissue O2 saturation response. Intensive Care Med. 2008;34:1600-7.

6. Donati A, Damiani E, Domizi R, Scorcella C, Carsetti A, Tondi S, et al. Nearinfrared spectroscopy for assessing tissue oxygenation and microvascular reactivity in critically ill patients: a prospective observational study. Crit Care 2016;20:311.

7. Neto AS, Pereira VG, Manetta JA, Espósito DC, Schultz MJ. Association between static and dynamic thenar near-infrared spectroscopy and mortality in patients with sepsis: a systematic review and meta-analysis. J Trauma Acute Care Surg. 2014;76:226-33.

8. De Blasi RA, Arcioni R, Brancadoro D, Rocco M. Effect of passive leg raising on microvascular venous compartment in critically ill patients. Minerva Anestesiol. 2016:82:1059-68.

9. Sanders J, Toor IS, Yurik TM, Keogh BE, Mythen M, Montgomery HE. Tissue oxygen saturation and outcome after cardiac surgery. Am J Crit Care. 2011 20:138-45.

10. Morel J, Bouchet JB, Vola M, Béraud AM, Clerc M, Awad S, et al. Tissue near infrared spectroscopy change is not correlated with patients' outcome in elective cardiac surgery. Acta Anaesthesiol Scand. 2014;58:835-42.

11. Tripodaki ES, Tasoulis A, Koliopoulou A, Vasileiadis I, Vastardis L, Giannis G, et al. Microcirculation and macrocirculation in cardiac surgical patients. Crit Care Res Pract. 2012;2012:654381

12. Kim TK, Cho YJ, Min JJ, Murkin JM, Bahk JH, Hong DM, et al. Microvascular reactivity and clinical outcomes in cardiac surgery. Crit Care. 2015;19:316.

13. Kopp R, Dommann K, Rossaint R, Schalte G, Grottke O, Spillner J, et al. Tissue oxygen saturation as an early indicator of delayed lactate clearance after cardiac surgery: a prospective observational study. BMC Anesthesiol. 2015;15:158.
14. Heller BJ, Deshpande P, Heller JA, McCormick P, Lin HM, Huang R, et al. Tissue oximetry during cardiac surgery and in the cardiac intensive care unit: a prospective observational trial. Ann Card Anaesth. 2018;21:371-5.

15. Butler E, Mahendran S, Nguyen J, Aneman A. Microvascular reactivity, assessed by near-infrared spectroscopy and a vascular occlusion test, is associated with patient outcomes following cardiac surgery: a prospective observational study. Eur J Anaesthesiol. 2018;35:356-64.

16. Biagioli B, Scolletta S, Cevenini G, Barbini E, Giomarelli P, Barbini P. A multivariate Bayesian model for assessing morbidity after coronary artery surgery. Crit Care. 2006;10(3):R94.

17. Ranucci M, Isgro G, Cazzaniga A, Ditta A, Boncilli A, Cotza M, et al. Different patterns of heparin resistance: therapeutic implications. Perfusion. 2002;17: 199-204.

18. Sakorafas $\mathrm{GH}$, Tsiotos $\mathrm{GG}$. Intra-abdominal complications after cardiac surgery. Eur J Surg. 1999;165(9):820-7.

19. Lipcsey M, Woinarski N.C, Bellomo R. Near infrared spectroscopy (NIRS) of the thenar eminence in anesthesia and intensive care. Ann Intensive Care 2012;2: 11.

20. Creteur J, Carollo T, Soldati G, Buchele G, de Backer D, Vincent JL. The prognostic value of muscle $\mathrm{StO}_{2}$ in septic patients. Intensive Care Med. 2007;33:1549-56.

21. Siebenmann C, Rasmussen P, Sorensen H, Zaar M, Hvidtfeldt M. Pichon a, et a. Card Output During Exerc: Comparison Four Methods Scand J Med Sci Sports. 2015;25:e20-7.

22. Mahutte CK, Jaffe MB, Chen PA, Sasse SA, Wong DH, Sassoon CS. Oxygen Fick and modified carbon dioxide Fick cardiac outputs. Crit Care Med. 1994; 22(1):86-95.

23. Giomarelli P, Scolletta S, Borrelli E, Biagioli B. Myocardial and lung injury after cardiopulmonary bypass: role of interleukin (IL)-10. Ann Thorac Surg. 2003;76:117-23.

24. Wan S, LeClerc JL, Vincent JL. Inflammatory response to cardiopulmonary bypass: mechanisms involved and possible therapeutic strategies. Chest. 1997:112:676-92.

25. Bakker J, Nijsten MW, Jansen TC. Clinical use of lactate monitoring in critically ill patients. Ann Intensive Care. 2013;10:3-12.

26. De Backer D, Ospina-Tascon G, Salgado D, Favory R, Creteur J, Vincent JL. Monitoring the microcirculation in the critically ill patient: current methods and future approaches. Intensive Care Med. 2010;36:1813-25.

27. Hernandez G, Bruhn A, Castro R, Regueira T. The holistic view on perfusion monitoring in septic shock. Curr Opin Crit Care. 2012;18:280-6.

28. Cossu AP, Suelzu S, Piu P, Orecchioni M, Bazzu G, Padua G, et al. Do on- and off-pump coronary bypass surgery differently affect perioperative peripheral tissue metabolism? Minerva Anestesiol. 2012;78:26-33.

29. De Backer D, Dubois MJ, Schmartz D, Koch M, Ducart A, Barvais L, et al. Microcirculatory alterations in cardiac surgery: effects of cardiopulmonary bypass and anesthesia. Ann Thorac Surg. 2009:88:1396-403.

30. Den Uil CA, Lagrand WK, Spronk PE, van Domburg RT, Hofland J, Luthen $C$, et al. Impaired sublingual microvascular perfusion during surgery with cardiopulmonary bypass: a pilot study. J Thorac Cardiovasc Surg. 2008;136:129-34.

31. Bauer A, Kofler S, Thiel M, Eifert S, Christ F. Monitoring of the sublingual microcirculation in cardiac surgery using orthogonal polarization spectral imaging: preliminary results. Anesthesiol. 2007;107:939-45.

32. Smith RS, Murkin JM. A novel assessment of peripheral tissue microcirculatory vasoreactivity using vascular occlusion testing during cardiopulmonary bypass. J Cardiothor Vasc Anesth. 2014;28:1217-20.

33. Bezemer R, Lima A, Myers D, Klijn E, Heger M, Goedhart PT, et al. Assessment of tissue oxygen saturation during a vascular occlusion test using near infrared spectroscopy: the role of probe spacing and measurement site studied in healthy volunteers. Crit Care. 2009;13:S4.

34. Monthé-Sagan K, Fischer MO, Saplacan V, Gerard JL, Hanouz JL, Fellahi JL. Near-infrared spectroscopy to assess microvascular dysfunction: a prospective pilot study in cardiac surgery patients. J Crit Care. 2016:31:264-8.

35. Butler E, Chin M, Aneman A. Peripheral near-infrared spectroscopy: Methodologic aspects and a systematic review in post-cardiac surgical patients. J Cardiothorac Vasc Anesth. 2016;S1053-0770(16):30299-3.

\section{Publisher's Note}

Springer Nature remains neutral with regard to jurisdictional claims in published maps and institutional affiliations. 